Mar. Biotechnol. 1, 1, 1999

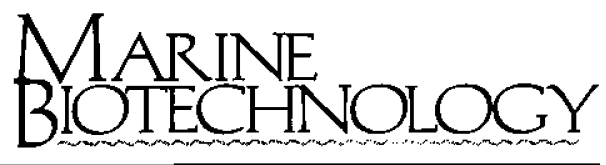

(๑) 1999 Springer-Verlag New York Inc.

\title{
EDITORIAL
}

\section{MARINE BIOTECHNOLOGY}

In the next millennium, the world's aquatic systems will be the new frontier for intensive biological investigation, resource development and management, and industrial application. To facilitate the development and expansion of this important frontier, a forum is needed for scientists to gather and exchange scientific information and ideas. Marine Biotechnology (MBT), incorporating Molecular Marine Biology and Biotechnology $(M M B B)$ and Journal of Marine Biotechnology $(J M B)$, is designed to fulfill this important need in the scientific community. This journal publishes a wide range of high-quality, peer-reviewed research articles that describe the use of biochemical, molecular, cellular, and immunochemical approaches to address fundamental questions and biotechnological applications of marine and freshwater organisms.

We will consider for publication articles describing the molecular biology, genetics, cell biology and biochemistry of any aquatic prokaryote or eukaryote. Papers on biotechnological applications should address fundamental biological questions or should demonstrate novel technical developments. We will also consider work on marine natural products when it meets these criteria. The journal will publish articles describing innovative molecular techniques for the study and manipulation (including transgenesis) of marine and freshwater organisms or research pertaining to industrial applications (aquaculture/mariculture) of aquatic organisms.

Under the resourceful leadership of Professors Demnis A. Powers, Shigetoh Miyachi, and Tadashi Matsunaga, papers published in $M M B B$ and $J M B$ achieved a high standard and excellent scientific quality. We thank them and the members of both editorial boards for their devotion. Furthermore, we also thank all those authors who submitted their high-quality scientific papers to both journals. Without the tireless efforts of editor-inchief, editorial board members, reviewers, and authors, it would have been impossible for Marine Biotechnology to inherit such a strong foundation. We pledge to maintain and heighten the scientific quality of publications in Marine Biotechnology.

Thomas T. Chen

Yves LeGal

Shigetoh Miyachi 